\title{
Friction and Wear of Mineral Surfaces in Liquid Environments
}

\author{
Carlos M. Pina, Carlos Pimentel and E. Gnecco
}

\begin{abstract}
Lateral Force Microscopy (LFM) is a very suitable technique to investigate the structure and reactivity of mineral surfaces in liquids. Studies performed in the last two decades have shown that the dissolution and growth of mineral surfaces immersed in water and aqueous solutions can be monitored by recording friction sig-nals with LFM. Moreover, the sensitivity of lateral forces to both structure and chem-istry makes possible to use LFM to obtain information about monolayers formed on mineral faces. Finally, numerous mineral surfaces are excellent substrates on which nanoparticles and complex organic molecules can be deposited and subsequently imaged and manipulated. This opens the way to future applications in molecular electronics. This chapter presents an overview of the recent use of LFM in liquid to investigate mineral surfaces and processes occurring on them.
\end{abstract}

\subsection{Introduction}

Minerals have been revealed as an almost inexhaustible source of surfaces which can be studied with atomic force microscopy (AFM). As early as 1992, the (104) surface of the mineral calcite was chosen by several researchers to conduct first investigations of a relatively complex crystal face using AFM [1-3]. Since then, mineral surfaces have been extensively used to investigate the structure, reactivity (e.g. growth and

C.M. Pina C . Pimentel

Departamento de Cristalografía y Mineralogía, Universidad Complutense de Madrid, Instituto de Geociencias (UCM-CSIC), c/José Antonio Novais, 2, 28040 Madrid,

Spain e-mail: cmpina@geo.ucm.es

C. Pimentel

e-mail:

cpimentelguerra@geo.ucm.es

E. Gnecco

Instituto Madrileño de Estudios Avanzados en Nanociencia, IMDEA Nanociencia,

Campus Universitario de Cantoblanco, 28049 Madrid, Spain

e-mail: enrico.gnecco@imdea.org 
dissolution), mechanical properties and wearing of crystalline matter [4-6, and references therein]. Furthermore, numerous mineral surfaces are suitable substrates to perform nanomanipulation experiments in which overgrowths, nanoparticles and complex biomolecules are imaged, disrupted, removed or displaced [7-9]. However, to obtain reliable quantitative information from these experiments it is essential to measure and control both vertical (loading) and lateral (friction) forces between the tip of the AFM and the investigated surfaces. When an AFM is used to record fric-tion forces as a function of loading forces it is usually called lateral force microscope (LFM) or friction force microscope (FFM) [10]. Since recorded friction forces are sensitive to both the composition and structure of the substrates and the bonds formed between them and the scanning tip, LFM can, in principle, provide information about the crystallochemistry of surfaces. This is why LFM is also sometimes named chem-ical force microscopy (CFM), despite quantitative chemical analysis of surfaces is still beyond the current capabilities of this technique. The main obstacle to extract reliable structural and chemical information of surfaces from recorded lateral forces is the high complexity of the interactions between the tip probe and the surfaces. When an AFM tip slides on a surface, measured friction forces are influenced by a high number of factors, such as the elastic properties of both tip and surface, the tip-surface contact area, the sliding velocity, the formation and breaking of (chemi-cal) bonds between the tip apex and the atoms of the surface, and the capillarity and adhesion forces. To reduce the number of factors affecting frictional forces and to increase the reproducibility of friction data, researchers perform their measurements using AFM tips with well characterised sizes, geometries and elastic properties, and operating under controlled conditions. The optimum controlled conditions are ultra-high vacuum (UHV) and/or low temperatures. This decreases the concentration of impurities, eliminates the adhesion forces between tip and surfaces due to humidity, and reduces the thermal vibration of atoms, which negatively affects the quality of the friction data. These advantages are, however, compromised by the demanding technical requirements of LFM in UHV. Moreover, relevant studies on the reactivity and frictional properties of mineral surfaces usually require observational conditions similar to those found in natural environments and, among them, liquid environments (i.e. aqueous solutions) are doubtless the most important.

The nanotribological investigation of mineral surfaces in liquid environments constitutes a new research field. Both the measurement of friction and nanomanipulation of molecules, particles or overgrowths on minerals immersed in liquids (e.g. water, aqueous solutions, and organic liquids such as ethanol) are not only of interest because natural environments and processes can be reproduced but also because it has been shown that LFM microscopy in liquids is can be an alternative to other AFM techniques in UHV which provide images with comparable resolution.

In this chapter, we review recent nanotribological investigations of mineral surfaces immersed in liquids. This review will show that LFM operating in a liquid environment is a very suitable technique to obtain structural and, to some extent, chemical information of crystal surfaces. Furthermore, it will be shown that the friction maps acquired during the interaction of mineral surfaces with liquids can be used to study mineral reactivity. Finally, recent investigations demonstrate that 
mineral surfaces can act as adequate substrates to deposit organic molecules. The subsequent nanomanipulation of such molecules can provide new insights into the processes that control the interaction between inorganic and organic matter.

\section{Structural Studies of Mineral Surfaces Using Lateral Force Microscopy}

Lateral force microscopy can be used to investigate structural details of surfaces which are impossible or extremely difficult to resolve using conventional AFM. For instance, it has been demonstrated that recorded friction signals are sensitive to the orientation of some ionic groups (e.g. sulphate and carbonate groups) protruding from cleavage mineral surfaces $[11,12]$. On a number of surfaces of alkaline earth sulphate crystals, i.e. anhydrite (100), barite (001) and celestine (001), Shindo et al. [11] measured an alternation of friction values in successive crystal monolayers. While the friction contrast can be attributed to the relative orientation of the S-O bonds of sulphate groups with respect to the scan direction, its alternation is due to the reversal of such an orientation by the operation of two-fold screw axes perpendicular to the studied mineral surfaces, which rotate $180^{\circ}$ the position of the sulphate groups in successive monolayers (see Fig. 9.1). In the case of the three sulphates investigated, the higher friction was found when the monolayers were scanned against the tilt of the sulphate groups.

Kwak and Shindo [12] also studied the frictional asymmetry due to the tilt of carbonate groups on calcite $\{104\}$ surfaces [12]. To this end, these authors scanned calcite (104) surfaces of two crystals oriented opposite. Although again different friction values were found depending on the scan direction, a lower friction was

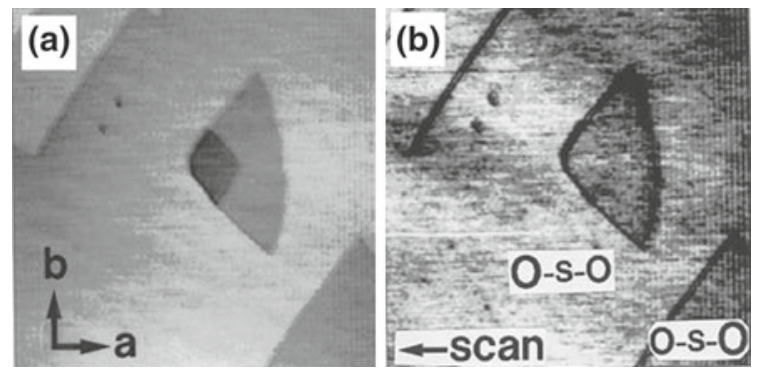

Fig. 9.1 a Topography AFM image of a celestine (001) surface showing a triangular etch pit with a rhombus-shaped pit inside. The depth of both etch pits is $0.34 \mathrm{~nm}$, i.e half a unit cell each. b LFM image of the same area showing the reversal of friction in successive monolayers. Larger "O" symbols indicate the position of the protruding oxygens belonging to the sulphate groups. With the set up used, dark areas indicate higher friction. Scan area: $1.46 \times 1.46 \mu \mathrm{m}^{2}$. Both images were taken with a scan direction from the right to the left. Reprinted (adapted) with permission from Shindo et al. [11]. Evidence of the contribution of molecular orientations on the surface force friction of alkaline earth sulfate crystals. Phys. Chem. Chem. Phys. 1, 1597-1600 Copyright 1999 PCCP 
measured when scanning against the tilt of the carbonate groups. This result contrasts with the tribological behaviour previously observed on sulphates. An explanation for such a discrepancy can be found in the different tilt angles of the $\mathrm{C}-\mathrm{O}$ and $\mathrm{S}-\mathrm{O}$ bonds with respect to the surfaces, which result in different elastic responses when the tip is pushing down the $\mathrm{CO}_{3}$ and $\mathrm{SO}_{4}$ groups during the scan. Although a conclusive explanation for the reversed friction anisotropy observed on faces of alkali earth sulphates and calcite requires further experimental and modelling work, the current observations clearly show that the interpretation of friction contrast in terms of molecular orientations is not straightforward.

The effects of molecular orientations on friction were observed by Shindo and collaborators on mineral surfaces exposed to air. Nanotribological studies of mineral surfaces immersed in water have provided similar results. For example, in situ observations of crystal growth on anhydrite (100) surfaces from aqueous solutions have shown that friction contrast reverses in successive monolayers during growth [13] (see Fig. 9.2). Such a friction contrast reversal can be again explained as due to the alternate orientation of sulphate groups within elementary growth layers related by two-fold screw axes.

LFM imaging in water is also useful to obtain information of slight structural modifications of mineral surfaces like calcite (104) and dolomite (104) faces. In first high resolution AFM studies of calcite (104) surfaces conducted in water, a rectangular lattice consistent with the calcite bulk structure was reported [1, 2, 14-16]. In addition, AFM images showed two peculiarities in the termination of the calcite (104) face: (i) the existence of rows of atoms parallel to the [010] direction which alternate in height and they are paired, i.e. the so-called "row-pairing"; and (ii) the height modulation along the direction [010] with a periodicity of about $1 \mathrm{~nm}$ which results in a $(2 \times 1)$ reconstruction of the calcite $(104)$ surface $[15,16]$. Both the rowpairing and $(2 \times 1)$ reconstructions have been confirmed recently by non-contact AFM in water and in UHV [4, 17, and references therein].
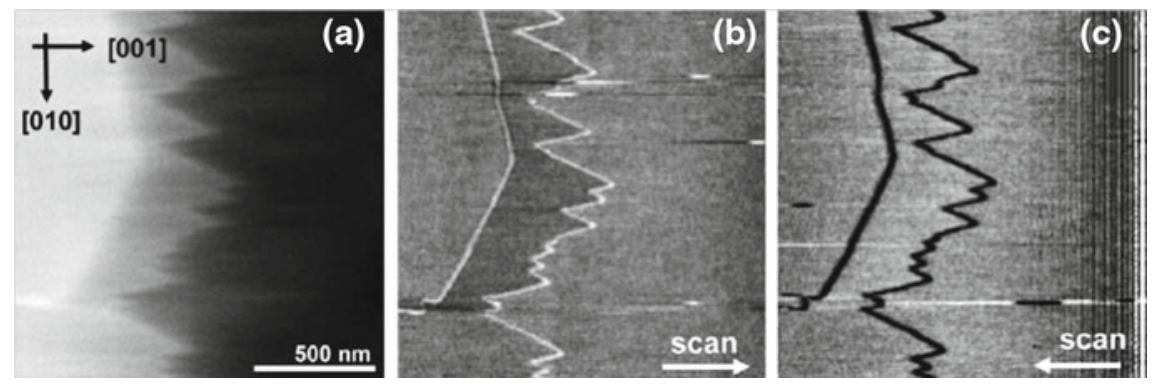

Fig. 9.2 a Topography image showing the growth of a monolayer on an anhydrite surface. b, c LFM images of the same area taken with opposite scan directions and showing the reversal of friction contrast. Reprinted (adapted) with permission from Pina [13]. Nanoscale dissolution and growth on anhydrite cleavage faces. Geochimica et Cosmochimica Acta 73, 7034-7044. Copyright 2009 Elsevier 
High resolution LFM images of the (104) faces of both calcite and the structurallyrelated mineral dolomite, $\mathrm{CaMg}\left(\mathrm{CO}_{3}\right) 2$, have provided further information about their surface structures and reconstruction phenomena described above. Friction maps of calcite and dolomite (104) surfaces in water show two distinct friction peaks in each rectangular surface unit cell, which can be univocally attributed to the interaction of the AFM tip with the protruding oxygens of the triangular carbonate groups (see Fig. 9.3).

A more detailed analysis of the friction forces during the scan of calcite and dolomite (104) demonstrated that they depend on the scan direction, i.e. there is a clear anisotropy of friction [18]. This is evidenced by the weakening of the intensity of one of the friction peaks within the dolomite and calcite surface unit cells when the scan direction approaches the [010] crystallographic direction (see Fig. 9.4). In addition, high resolution LFM images of dolomite and calcite (104) surfaces in water confirmed the row-pairing previously observed using other AFM modes (i.e. topography and deflection). By changing the scan direction LFM images also show for the first time an inversion of the row-pairing. However, up to date, high resolution LFM images of both dolomite and calcite (104) surfaces do not show evidences of the $(2 \times 1)$ reconstruction.

The reported atomic scale friction maps of dolomite and calcite (104) surfaces, as well as their modifications with the scan direction, have been adequately reproduced by numeric calculations. Lattice resolved friction force maps like those shown in Figs. 9.3 and 9.4 can be understood using the Prandtl-Tomlinson model [18]. Here, specific chemical groups in the unit cell of the crystal surface act as pinning centers for the AFM tip. In this case, the lateral force exerted on the tip increases linearly

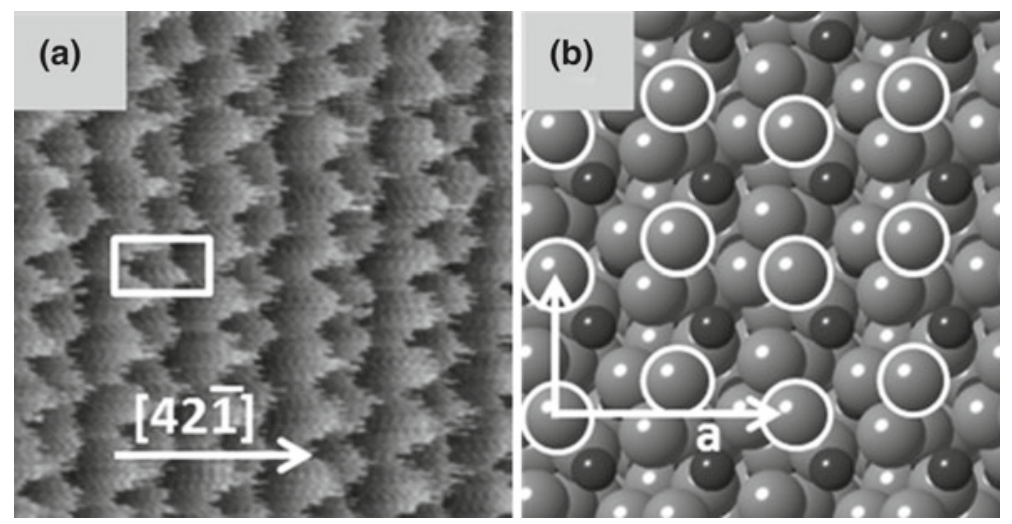

Fig. 9.3 a Friction map of a calcite (104) face showing two friction peaks per unit cell (marked by a white rectangle). b Projection of the calcite structure on the (104) plane. Oxygen atoms are represented by light grey spheres, carbon atoms by black spheres, and calcium atoms by dark grey spheres. Carbon atoms are not visible in this projection. Protruding oxygens atoms from the surface are enhanced with a white rim. In the dolomite structure, half of the $\mathrm{Ca}$ atoms are replaced by $\mathrm{Mg}$ along the [421] direction, the surface structure being essentially identical. Reprinted (adapted) with permission from Pina et al. [18]. Anisotropic coupling while sliding on dolomite and calcite crystals. Physical Review B, 85, 073402 

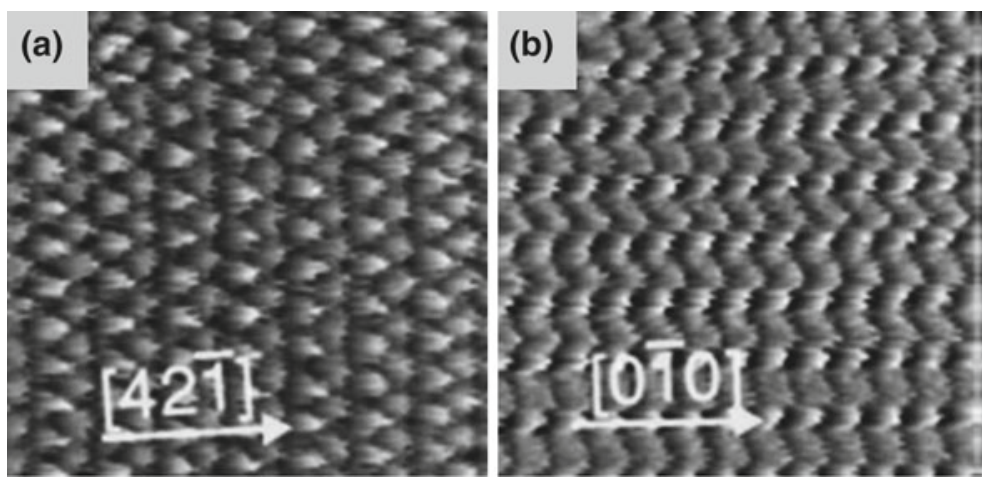

Fig. 9.4 Friction maps of calcite (104) surface when scanning almost parallel to (a) th e [ $\left.\begin{array}{lll}4 & 2 & 1\end{array}\right]$ direction and the, (b) the [ 0 10] direction. Reprinted (adapted) with permission from Pina et al. [18]. Anisotropic coupling while sliding on dolomite and calcite crystals. Physical Review B, 85, 073402

when the tip is pulled laterally away from the pinning center, as if an elastic spring was present between tip and surface. When the elongation of the spring (and the corresponding lateral force) reaches a critical value, pinning is no more sustainable and the tip suddenly jumps into a new pinning site defined by the scan direction. In this context, we should notice that, unless isolated defects or consecutive atoms below and above a step edges are imaged, it is not correct to speak about true molecular resolution. The number of atoms forming the contact area can indeed be in the order of some tens, so that the contrast seen in the friction force maps is ultimately due to the convolution of the real crystal structure with the atomic arrangement at the tip apex.

\section{Obtaining Chemical Information of Surfaces from Frictional Forces}

On atomically flat surfaces, frictional contrast can be related to compositional contrast [19]. This enables the in situ observation of adsorption and/or solid solution formation on mineral surfaces from multicomponent aqueous solutions, which is fundamen-tal to better understand a number of mechanisms that control the removal of some contaminants from natural and industrial waters. The first systematic nanotribological study of the interaction of dissolved metal ions with a mineral surface was conducted by Hay et al. [19]. These authors observed with LFM the reactions between calcite (104) surfaces and aqueous solutions containing $\mathrm{Ca}^{2+}, \mathrm{Sr}^{2+}$ and $\mathrm{La}^{3+}$. Such reac-tions led to the formation of monolayer overgrowths which were identified by clear differences in the recorded friction signals (Fig. 9.5). In the three cases, newly-grown layers display higher friction contrast than the calcite substrate, which was attributed to epitaxial strain. Differently, epitaxial monolayers of celestine and anglesite grown on barite (001) have been found to show lower friction than the substrate [20]. 
Topography
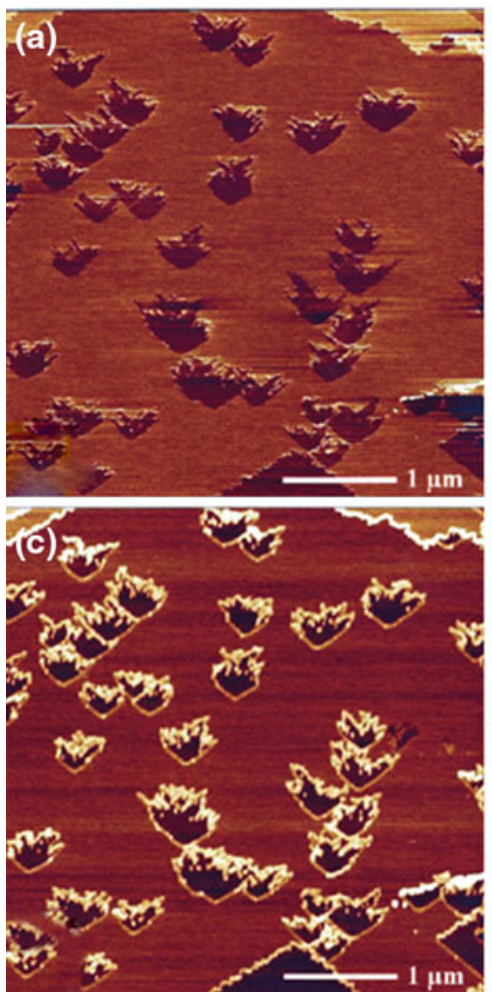

Friction
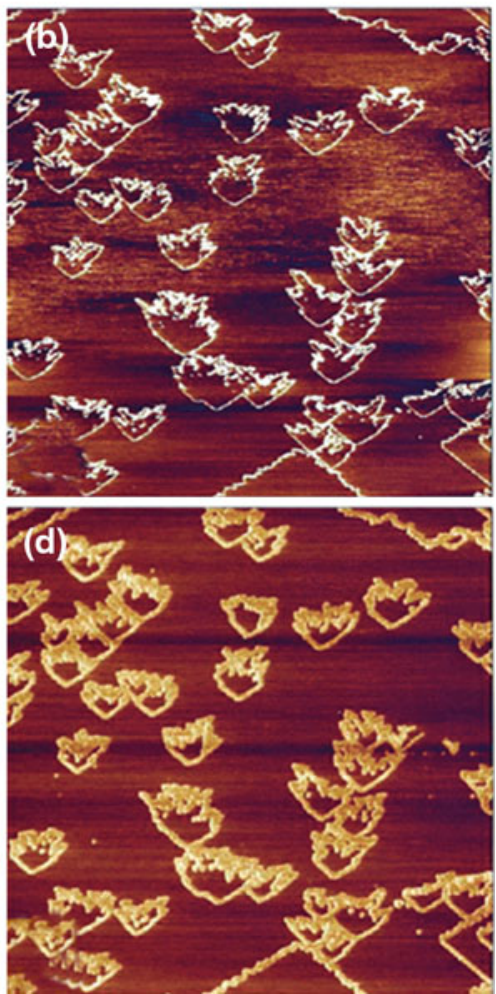

Fig. 9.5 Topography and friction images of a growing calcite (104) surface in the presence of $\mathrm{La}^{3+}$. a and $\mathbf{b}$ after $150 \mathrm{~s}$ of reaction. $\mathbf{c}$ and $\mathbf{d}$ after $1330 \mathrm{~s}$ of reaction. Higher contrast areas in the friction images correspond to the places where a La-carbonate nucleated. Reprinted (adapted) with permission from Hay et al. [19]. Mechanisms of metal ion sorption on calcite: composition mapping by lateral force microscopy. Langmuir, 19, 3727-3740. Copyright 2003 American Chemical Society

These somehow contradictory experimental results indicate that the origin of friction when epitaxial layers are formed is not clear and further experimental and computational work is required.

Despite the current limitations of the use of LFM to detect chemical changes on mineral surfaces and overgrowths, the study of friction have already provided semiquantitative information about the up-take of dissolved metals by common mineral surfaces and its effect on the nanotribological properties of the overgrowths. An interesting case study, the formation of Cd-bearing carbonate layers on calcite, was recently investigated with LFM [21]. Systematic measurements of friction versus applied loading force were conducted both on calcite and $\mathrm{Cd}$-bearing carbonate layers and successfully fitted to the Johnson-Kendall-Roberts model, which accounts for adhesion in single asperity contacts [22] ( s e e F i g . 9.6a). According to this model, differences in friction between calcite substrate and Cd-bearing layers have a different origin depending on the loading force. At low loading forces, differences in friction 


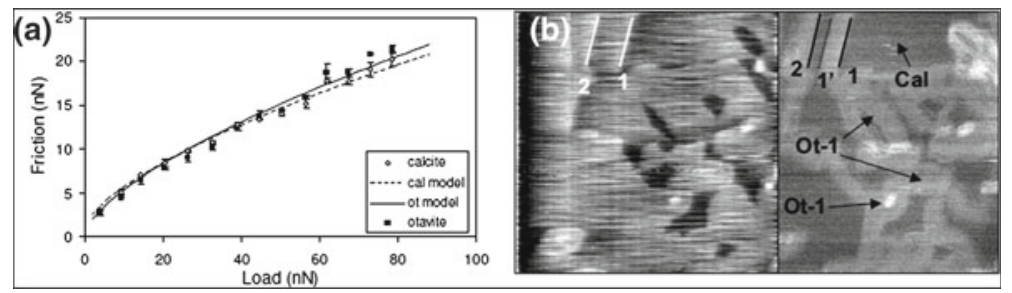

Fig. 9.6 a Friction versus loading force measured on calcite substrate and otavite overgrowths. b Overgrowth of otavite (Ot-1) on calcite (104) surface (Cal). Left topography image; Right friction image. Modified from Cubillas and Higgins [21]. Friction characteristics of Cd-rich carbonate films on calcite surfaces: implications for compositional differentiation at the nanometer scale. Geochemical Transactions 10.7. doi:1186/1467-4866-10-7

are determined by adhesion forces. Differently, at high loading forces, friction forces are mainly sensitive to differences in contact shear strengths. As a result, while at low loading forces (up to $40 \mathrm{nN}$ ), Cd-bearing layers have lower friction than the calcite substrate, at loading forces higher than approximately $40 \mathrm{nN}$, friction measured on calcite substrates is lower than that on Cd-bearing layers (see Fig.9.6b).

The results summarized above, although somehow disappointing, clearly demonstrate that future quantifications of chemical variations on mineral surfaces using LFM will require a precise knowledge of the regime (i.e. adhesion-controlled or shear strength-controlled) under which friction is measured.

\subsection{Wear and Nanomanipulation of Mineral Surfaces and Overgrowths}

AFM can be used not only to image but also to produce wear and reorganisation of mineral surfaces at the nanoscale under different environmental conditions including aqueous solutions. In addition, overgrowths formed on mineral surfaces can be eroded and/or manipulated using the tip of the AFM. Such wear and nanomanipulation experiments are conducted by controlling the loading forces of the AFM tip and they provide information about the mechanical properties and stability of both mineral surfaces and mineral overgrowths. Furthermore, nanowear experiments allow one to study phenomena such as the propagation of ductile and brittle deformation, stressenhanced crystal growth and dissolution and surface pattern formation (e.g. ripple initiation and propagation [23]).

Nanowear experiments conducted on calcite $\left(\mathrm{CaCO}_{3}\right)\{104\}$ faces and brushite $\left(\mathrm{CaHPO}_{4}-2 \mathrm{H}_{2} \mathrm{O}\right)\{010\}$ faces immersed in undersaturated aqueous solutions have demonstrated that when vertical forces are increased dissolution behaviour is strongly modified [24, 25]. On these faces, it was observed that moderate to high loading forces produce relatively atomically flat surfaces. This surface planarization, also observed on dolomite (104) and gypsum $\left(\mathrm{CaSO}_{4} \cdot 2 \mathrm{H}_{2} \mathrm{O}\right)(010)$ surfaces, can be explained by a combination of enhanced dissolution of pre-existent steps and etch pits edges with a tip assisted redeposition of dissolved material during scan. In contrast, redeposition 
outside the scan areas lead to an increase in roughness, further indicating the strong effect of the AFM tip on the surface reorganisation.

When supersaturated solutions with respect to calcite are used, crystal growth is enhanced by the AFM tip along pre-existing steps, even at relatively low loading forces. Such a growth enhancement rapidly increases with supersaturation. The result of this tip-enhanced growth is the formation of defect-free calcite (104) surfaces at high velocities [26]. To promote such a high growth rates in the absence of scanning higher supersaturations with respect to calcite are required, which however do not lead to a rapid layer-by-layer material deposition but to a three-dimensional nucleation on calcite surfaces. As in the case of dissolution, tip-enhanced growth can be explained by the mobilisation of ions and clusters of ions during scan (see Fig. 9.7). In particular, the AFM tip seems to have the ability of moving growth units from calcite terraces to the proximity of step edges where edge diffusion occurs. Then growth units are eventually incorporated into the calcite structure.

The tip of the AFM can be used not only to modify the dissolution and growth behaviour of mineral surfaces but also to remove overgrowths previously formed on them. When such nanomanipulation experiments are conducted with controlled loading forces and by recording friction forces, quantitative information on the stability of overgrowths on mineral substrates can be obtained. Two interesting model examples for studying the stability of overgrowths on mineral surfaces are the formation of calcite islands on dolomite and the isostructural kutnohorite $\left(\mathrm{MnCa}\left(\mathrm{CO}_{3}\right)_{2}\right)\{104\}$ surfaces [7]. At high supersaturations with respect to calcite, spontaneous growth of calcite three-dimensional islands on dolomite and kutnohorite can be observed. This growth is in both cases epitaxial and calcite $\{104\}$ rhombohedra are deposited on the dolomite and kutnohorite substrates with an almost perfect parallelism of the [42 $\overline{1}]$ and [010] crystallographic directions, i.e. the main directions lying on the (104) epitaxial contact plane (see Fig. 9.8).

Calcite islands grown on dolomite and kutnohorite (104) surfaces can be removed by increasing the loading force of the AFM. Figure 9.9a, b shows a typical event of island detachment in which a single island and an aggregate of two calcite crystals were removed from a dolomite (104) surface by the AFM tip. As can be seen in Fig. 9.9c the removal of each calcite island results in a sharp increase in the recorded friction force. From such frictional peaks, estimations of the shear strengths required to remove epitaxial overgrowths can be obtained using the simple formula $\tau=F_{L} / A$ where $F_{L}$ is the lateral force measured at moment and $A$ is the overgrowth-substrate contact area, which can be measured from topography images. First nanomanipulation experiments provided the following shear strengths: $\tau \approx 7 \mathrm{MPa}$ for calcite islands grown on dolomite (104) surface and $\tau \approx 140 \mathrm{MPa}$ for calcite islands grown on kutnohorite (104) surface. The significant difference in shear strengths can be partially explained by the differences in lattice misfits between calcite overgrowth and substrates (which are higher in the case of the dolomite (104) substrate).

Recent nanomanipulation experiments have been also addressed to remove nitratine $\left(\mathrm{NaNO}_{3}\right)$ islands grown on calcite (104) surfaces [8]. Nitratine and calcite are isostructural compounds and as in the case of calcite on dolomite and kutnohorite substrates, epitaxial growth occurs. Nanomanipulation experiments, similar to those 
Fig. 9.7 Growth within an etch pit two monolayers deep on a calcite (104) surface. The growth occurred from a slightly supersaturated aqueous solution with respect to calcite and applying a tip loading force $F_{N}=7 \mathrm{nN}$. The crystallographic directions of the steps edges that define the etch pit are indicated by solid lines. The dashed line mark the initial shape of the etch pit. Reprinted (adapted) with permission from McEvoy et al. [26]. Scanning-Induced Growth on Single Crystal Calcite with an Atomic Force Microscope. Langmuir 22, 6931-6938. Copyright 2006 American Chemical Society
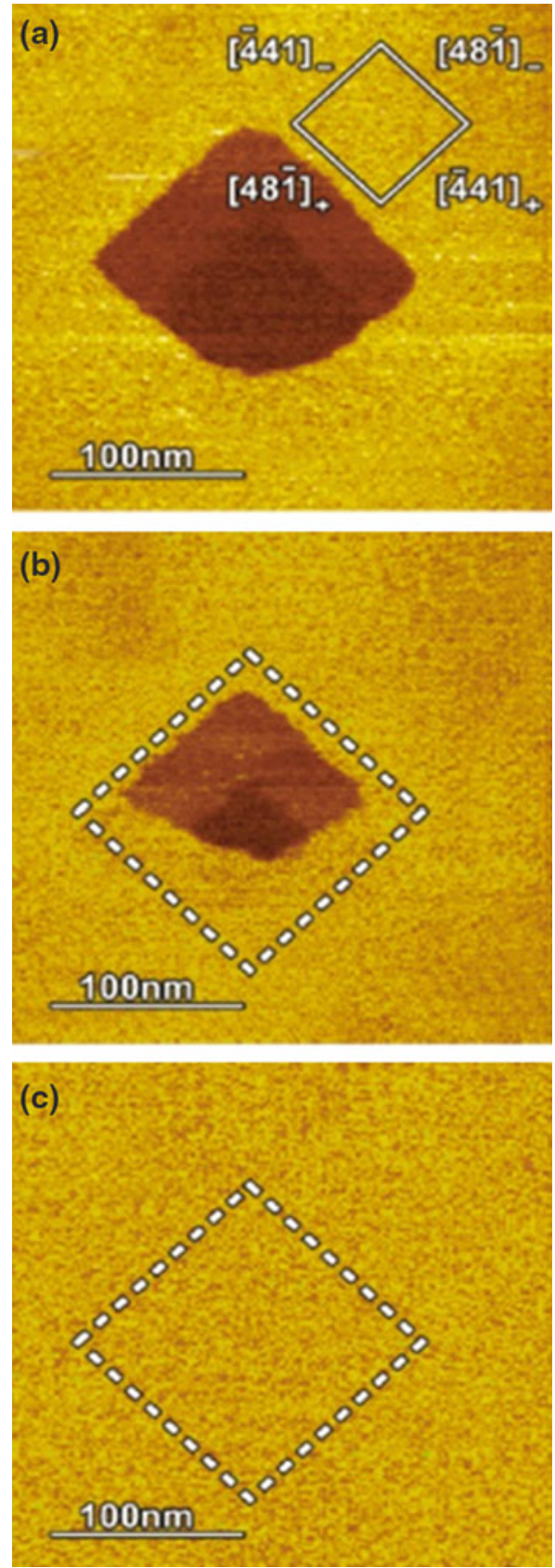




Fig. 9.8 AFM deflection images showing the epitaxial growth of calcite islands on the (10.4) surfaces of (a) dolomite (scan area: $14 \times 14 \mu \mathrm{m}^{2}$ ) and (b) kutnohorite (scan area: $5 \times 5 \mu \mathrm{m}^{2}$ ). Main crystallographic directions are indicated by white arrows. Reprinted (adapted) with permission from Pimentel et al. [7]. Epitaxial growth of calcite crystals on dolomite and kutnohorite (104) surfaces. Crystal Growth and Design 13, 2557-2563. Copyright 2013 American Chemical Society
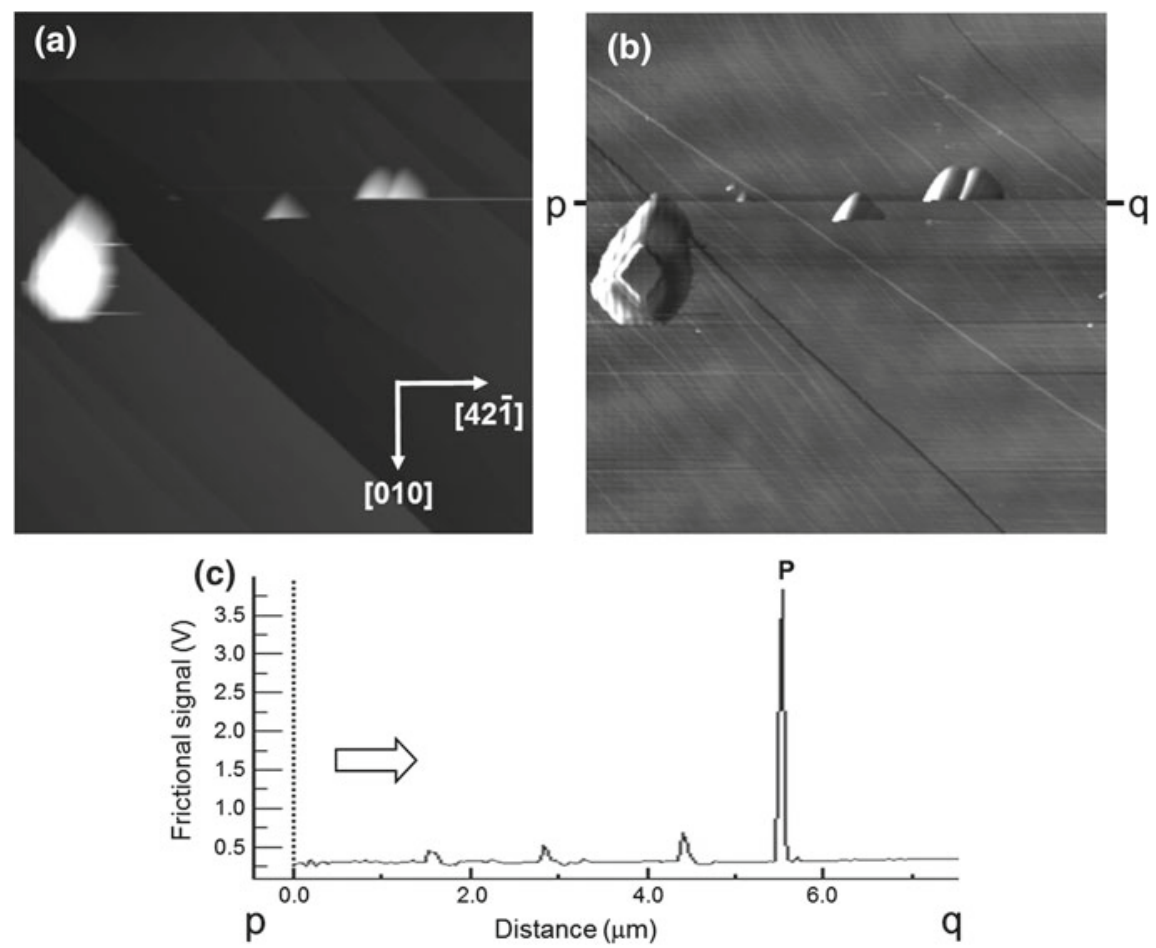

Fig. 9.9 Removal of calcite islands grown on a dolomite (104) surface. a Topography AFM image. b Friction AFM image of the same area. Both images are $7.5 \times 7.5 \mu \mathrm{m}^{2}$ in size. $\mathbf{c}$ Friction profile taken along the $\mathrm{p}$-q line in (b). The frictional peak $(P)$ corresponds to the removal of the aggregate of two calcite islands in (b). The arrow indicates the scan direction. Reprinted (adapted) with permission from Pimentel et al. [7]. Epitaxial growth of calcite crystals on dolomite and kutnohorite (104) surfaces. Crystal Growth and Design 13, 2557-2563. Copyright 2013 American Chemical Society 

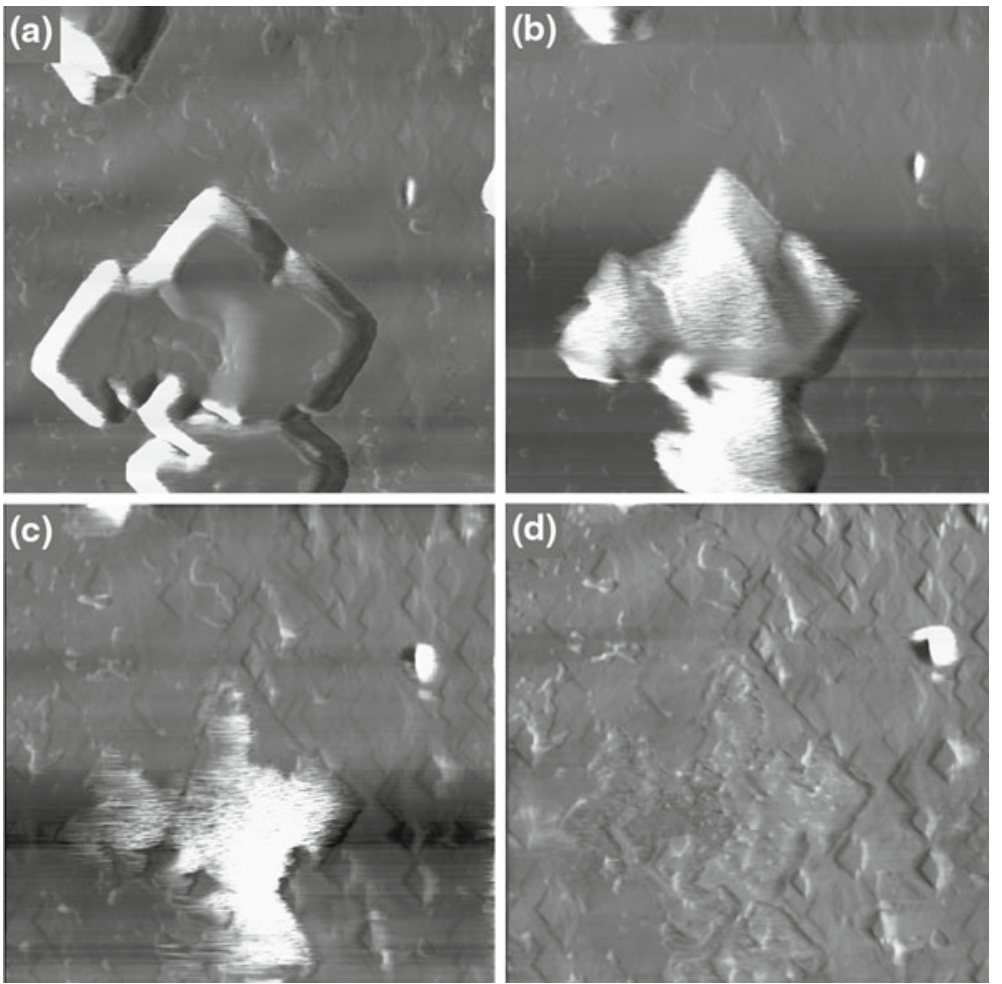

Fig. 9.10 Sequence of AFM friction images showing the nanowear of nitratine islands grown on a calcite (104) face. The applied normal force was $F_{N} \approx 80 \mu \mathrm{N}$. Reprinted (adapted) with permission from Benages-Vilau et al. [8]. Epitaxial crystal growth of nitratine on calcite (10.4) cleavage faces at nanoscale. Crystal Growth and Design 13,5397-5403. Copyright 2013 American Chemical Society

conducted in the case of calcite islands on dolomite and kutnohorite (104) surfaces, provided estimates of shear strengths required to remove epitaxial overgrowths of about $30 \mathrm{MPa}$. However, due to the softness of nitratine islands (1.5-2 in the Mohs scale), the removal of nitratine islands is often accompanied by their erosion, which is also an example of nanowear under controlled conditions (Fig. 9.10).

\subsection{Organic Molecules on Mineral Surfaces}

Mineral surfaces provide a number of substrates on which both organic molecules can be deposited and subsequently nanomanipulated. This kind of experiments are of interest for various research and industrial fields such as the production of new composite materials, the studies of biomineralisation mechanisms and the design of new insulating and semiconductor materials. Insulating mineral surfaces are very 
promising materials in the context of molecular electronics. Here, the minerals can be used as substrates for growing self-assembled structures of organic molecules, which need to be electrically decoupled from the surrounding environment. A thick insulating substrate makes the use of scanning tunneling microscopy as direct imaging technique, clearly impossible. On the other side, the AFM can still provide detailed information on the morphological structures of the organic layers so formed. The organic molecules are usually deposited onto the substrate in ultra-high vacuum, and observed by AFM in the same environment. For some recent examples we refer the reader to a recent review by Rahe et al. [27]. Nevertheless, an accurate resolution is also possible in different contexts, which better reproduce the conditions under which the molecular devices are supposed to be operated.

Figure 9.11 shows a topographic image of dolomite (104) covered by about 2 monolayers of copper phthalocyanine ( $\mathrm{CuPc}$ ) molecules [28]. Elongated molecular stripes with height of about $4.5 \mathrm{~nm}$ are seen all over the surface. The width of the stripes varies between 200 and $600 \mathrm{~nm}$, and their length is about $1-3 \mu \mathrm{m}$. The stripes do not reveal any preferential orientation, meaning that the intermolecular interactions are much stronger than the molecule-substrate interaction.

Some features in the internal structure of the molecular stripes could be resolved by friction force microcopy, as shown in Fig.9.12a. Here, a stacked intermolecular arrangement can be recognized. The stacks run along the axis of the corresponding stripe and, consequently, with no preferential orientation with respect to the substrate.

Fig. 9.11 a Contact mode AFM topography $\left(4.6 \times 6 \mathrm{~nm}^{2}\right)$ of the dolomite (104) surface covered by 2.0 monolayers of $\mathrm{CuPc}$. The image was acquired at room temperature in water. $\mathbf{b}$ Topography profile taken along the continuous line in (a). Reprinted from Nita et al. [28]. Molecular resolution friction microscopy of $\mathrm{Cu}$ phthalocyanine thin films on dolomite (104) in water. Nanoscale 6, 8334-8339. Copyright 2014 The Royal Society of Chemistry



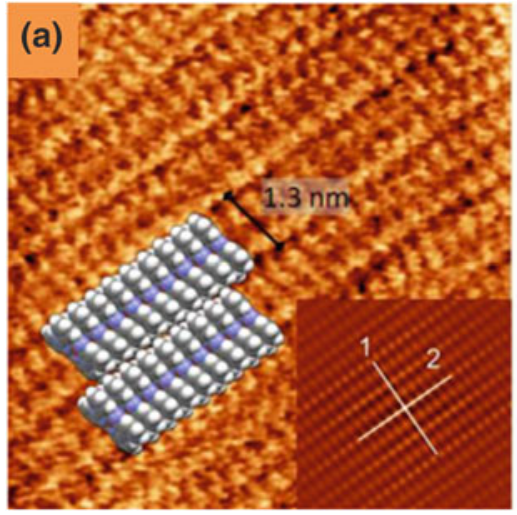

(b)

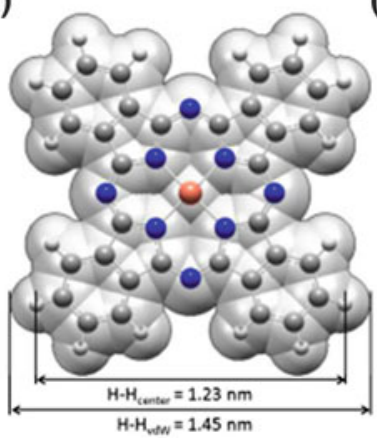

(c)

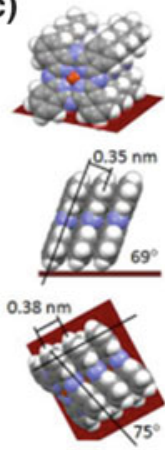

Fig. 9.12 a High resolution friction map $\left(10 \times 10 \mathrm{~nm}^{2}\right)$ on a CuPc stripe. The periodic arrangement of the molecules in parallel stacks, running along the axis of the stripe, is clearly visible. The average friction force is $5.5 \mathrm{nN}$ (corresponding to a normal force $F_{N}=2.7 \mathrm{nN}$ ). Inset shows a 2D self-correlation of the friction map. b Chemical structure of a copper (II) phthalocyanine (CuPc) molecule. $\mathbf{c}$ Molecular stack geometry as determined from single crystal x-ray diffraction. Reprinted from Nita et al. [28]. Molecular resolution friction microscopy of $\mathrm{Cu}$ phthalocyanine thin films on dolomite (10.4) in water. Nanoscale 6, 8334-8339. Copyright 2014 The Royal Society of Chemistry

Two elongated spots per molecule are identified. Intermolecular structural parameters can be estimated from the 2D self-correlation analysis shown in the inset, which was performed along and perpendicular to the stacking direction. Along the stack, the periodicity is $0.36 \mathrm{~nm}$, close to the reported periodicity from the single crystal x-ray value $(0.38 \mathrm{~nm}$ ) (Fig. 9.12b). The measured stack width is $1.3 \mathrm{~nm}$, while the van der Waals (vdW) stack width calculated from single crystal data is $1.45 \mathrm{~nm} \times \sin 75^{\circ}=$ $1.40 \mathrm{~nm}$ (Fig. 9.12c). This indicates an interdigitated structure of neighboring $\mathrm{CuPc}$ stacks on dolomite just like in the single crystal which should effectively reduce the stack width by roughly the vdW radius of hydrogen $(0.11 \mathrm{~nm})$, resulting in a value of $1.29 \mathrm{~nm}$, in perfect agreement with the measured value. Accordingly, the crystal structure stack (projected against the stacking plane) can be perfectly overlaid with the FFM image, as shown in Fig.9.12a. In this way, the friction spots correspond to standing benzene rings. The good agreement of the stacking structure on dolomite with the single crystal data gives full evidence that substrate induction is weak on dolomite, and the growth of CuPc is essentially driven by self-assembly.

When the normal force increases, so do the pinning effect and the lateral force, and the resolution achieved via the stick-slip mechanism is enhanced. Nevertheless, this can also lead to irreversible damage of the molecular film. The image presented in Fig. 9.12a corresponds to a threshold value of $F_{L}=5.5 \mathrm{nN}$. If the same area is repeatedly scanned while keeping this value, the molecular layers are gradually worn off. This is seen in Fig. 9.13, where three 'snapshots' of the abrasive process at 

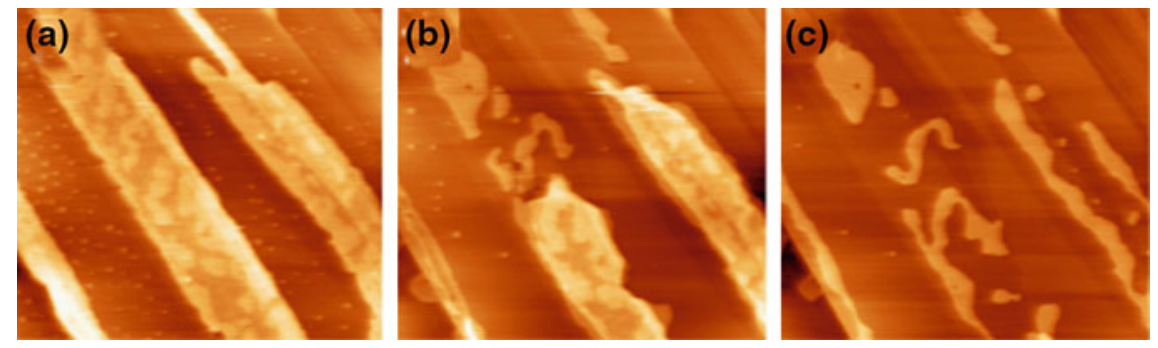

Fig. 9.13 Effect of prolonged scanning on the square region highlighted in Fig. 9.11. The topographic images $\mathbf{a}, \mathbf{b}$ and $\mathbf{c}$ were acquired during the $3 \mathrm{rd}, 19$ th and $22 \mathrm{nd}$ scan back and forth. Reprinted from Nita et al. [28]. Molecular resolution friction microscopy of $\mathrm{Cu}$ phthalocyanine thin films on dolomite (104) in water. Nanoscale 6, 8334-8339. Copyright 2014 The Royal Society of Chemistry

different times are shown. Two layers are progressively removed till the step edges of the underlying dolomite (104) surface become clearly visible in Fig. 9.13c. Note that, before being worn off, the molecular strips were running across the step edges of the substrate in a carpet-like fashion. This gives a third indication of the weak interaction between $\mathrm{CuPc}$ and the dolomite surface.

\subsection{Conclusions and Outlook}

To summarize, LFM in water is a promising new technique for obtaining high (atomic-scale) resolution images of both mineral and synthetic crystalline surfaces. Recent results demonstrate that the resolution and quality of the images achieved is comparable to those recorded using more demanding AFM techniques such as dynamic modes in UHV. This may be attributed to the absence of capillary effects in water, which reduces the adhesion between tip and surface and minimizes the damage while scanning. Analysis of friction maps at the nanoscale allows one to distinguish growth monolayers with different chemical composition. However, quantitative chemical analysis is still beyond the current capabilities of LFM. Nanomanipulation experiments where epitaxial overgrowths are removed from mineral surfaces can provide estimates of shear strengths, related to the adhesion forces between the overgrowths and substrates. Last but not least, the crystal structure of organic molecules self-assembled on the mineral surface can be also identified by FFM in water. The weak interaction between molecule and substrate observed in recent investigations on $\mathrm{CuPc}$ grown on dolomite (104) opens the way to interesting applications in molecular electronics. 
Acknowledgments This work was supported by the Spanish Ministerio de Economía y Competitividad (MINECO; project MAT2012-34487). Carlos Pimentel is grateful to Spanish Ministry of Education, Culture and Sports for a FPU grant. We thank Dr. Pawel Nita, Dr. Luo, Dr. MiliánMedina and Dr. Johannes Gierschner for their key collaboration on LFM of organic molecules on mineral surfaces.

\section{References}

1. P.E. Hillner, S. Manne, A.J. Gratz, P.K. Hansma, Ultramicroscopy 42-44, 1387 (1992). doi:10. 1016/0304-3991(92)90432-J

2. P.E. Hillner, A.J. Gratz, S. Manne, P.K. Hansma, Geology 20, 359 (1992). doi:10.1130/00917613(1992)020(lt)0359:ASIOCG(gt)2.3.CO;2

3. F. Ohnesorge, G. Binnig, Science 260, 1451 (1993). doi:10.1126/science.260.5113.1451

4. S. Rode, N. Oyabu, K. Kobayashi, H. Yamada, A. Kühnle, Langmuir 25, 2850 (2009). doi:10. $1021 / 1 \mathrm{a} 803448 \mathrm{v}$

5. I. Sethmann, J. Wang, U. Becker, A. Putnis, Cryst. Growth Des. 10, 4319 (2010). doi:10.1021/ $\operatorname{cg} 100202 \mathrm{~h}$

6. C.M. Pina, G. Jordan, in Nanoscopic Approaches in Earth and Planetary Sciences, ed. by F. Brenker, G. Jordan. EMU—Notes in Mineralogy (2006)

7. C. Pimentel, C.M. Pina, E. Gnecco, Cryst. Growth Des. 13, 2557 (2013). doi:10.1021/ $\operatorname{cg} 400315 \mathrm{~g}$

8. R. Benages-Vilau, T. Calvet, M.A. Cuevas-Diarte, C. Pimentel, C.M. Pina, Cryst. Growth Des. 13, 5397 (2013). doi:10.1021/cg401327h

9. S. Mangold, K. Harneit, T. Rohwerder, G. Claus, W. Sand, Appl. Environ. Microbiol. 74, 410 (2008). doi:10.1128/AEM.01812-07

10. E. Gnecco, R. Bennewitz, O. Pfeiffer, A. Socoliuc, E. Meyer, in Springer Handbook of Nanotechnology, ed. by B. Bhushan (Springer, Berlin, 2007)

11. H. Shindo, K. Shitagami, T. Sugai, S. Kondo, Phys. Chem.. Chem. Phys. 1, 1597 (1999). doi: 10. 1039/A808691E

12. M. Kwak, H. Shindo, Phys. Chem. Chem. Phys. 6, 129 (2004). doi:10.1039/B310991G

13. C.M. Pina, Geochimica et Cosmochimica Acta 73, 7034 (2009). doi:10.1016/j.gca.2009.08. 032

14. A.L. Rachlin, G.S. Henderson, M.C. Goh, Am. Mineral. 77, 904 (1992)

15. S.L.S. Stipp, C.M. Eggleston, B.S. Nielsen, Geochim. et Cosmochim. Acta 58, 3023 (1994). doi:10.1016/0016-7037(94)90176-7

16. S.L.S. Stipp, Geochim. et Cosmochim. Acta 63, 3121 (1999). doi:10.1016/S00167037(99)00239-2

17. P. Rahe, J. Schütte, A. Kühnle, J. Phys.: Condens. Matter 24, 084006 (2012). doi:10.1088/ 0953-8984/24/8/084006

18. C.M. Pina, R. Miranda, E. Gnecco, Phys. Rev. B 85, 073402 (2012). doi:10.1103/PhysRevB. 85.073402

19. M.B. Hay, R.K. Workman, S. Manne, Langmuir 19, 3727 (2003). doi:10.1021/la020647s

20. A.E. Murdaugh, M. Liddelow, A.M. Schmidt, S. Manne, Langmuir 23, 5852 (2007). doi:10. 1021/la063548d

21. P. Cubillas, S.R. Higgins, Geochem. Trans. 10, 7 (2009). doi:10.1186/1467-4866-10-7

22. K.L. Johnson, K. Kendall, A.D. Roberts, Proc. R. Soc. Lond. A 324, 301 (1971)

23. A. Socoliuc, E. Gnecco, R. Bennewitz, E. Meyer, Phys. Rev. B 68, 115416 (2003). doi:10. 1103/PhysRevB.68.115416

24. N.-S. Park, M.-W. Kim, S.C. Langford, J.T. Dickinson, J. Appl. Phys. 80, 2680 (1996). doi:10. $1063 / 1.363185$ 
25. L. Scudiero, S.C. Langford, J.T. Dickinson, Tribol. Lett. 6, 41 (1999). doi:10.1023/A: 1019134901387

26. A.L. McEvoy, F. Stevens, S.C. Langford, J.T. Dickinson, Langmuir 22, 6931 (2006). doi:10. 1021/la0608359

27. P. Rahe, M. Kittelmann, J.L. Neff, M. Nimmrich, M. Reichling, P. Maass, A. Kühnle, Adv. Mater. 25, 3948 (2013). doi:10.1002/adma.201300604

28. P. Nita, C. Pimentel, F. Luo, B. Milián-Medina, J. Gierschner, C.M. Pina, E. Gnecco, Nanoscale 6, 8334-8339 (2014). doi:10.1039/C4NR01522C 